\title{
Screening of $\mathbf{F}_{\mathbf{3}}$ Generation Interspecific Tomato Hybrids Derivatives for Resistance to Peanut Bud Necrosis Virus (PBNV)
}

\author{
Arumugam Nithyanandam ${ }^{1 *}$, A. Beaulah ${ }^{1}$, S. Harish ${ }^{2}$, \\ C. Rajamanickam ${ }^{1}$, S. Vellaikumar ${ }^{3}$ and P. Arunachalam ${ }^{4}$ \\ ${ }^{1}$ Department of Horticulture, ${ }^{2}$ Department of Plant Pathology, ${ }^{3}$ Department of Biotechnology, \\ ${ }^{4}$ Department of Plant Breeding and Genetics, Agricultural College \& Research Institute, \\ Tamil Nadu Agricultural University, Madurai- 625104, India \\ *Corresponding author
}

\section{A B S T R A C T}

Field screening of $\mathrm{F}_{3}$ generation interspecific tomato hybrids derived lines for resistance to Peanut bud necrosis virus (PBNV) was carried out in the Department of Horticulture, Agricultural College and Research Institute, Madurai, Tamil Nadu Agricultural University,

\section{Keywords}

Peanut bud necrosis virus, $\mathrm{PBNV}$, Resistance, Field screening, Percent Disease Incidence

\section{Article Info}

Accepted:

15 July 2020

Available Online:

10 August 2020

Tamil Nadu, during 2019-2020. PBNV is the most destructive and economically important plant virus in tomato which causes yield loss up to 80 to 100 per cent. The present study was formulated to develop PBNV resistant lines in tomato suitable for commercial cultivation. Totally 35 lines from four interspecific crosses Viz., 8 lines from H4 - Arka Vikas x PSR11668 (S. pimpinellifolium), 10 lines from H6 - Arka Vikas x EC519809 (S. peruvianum), 8 lines from H17 - CO3 x EC519791 (S. habrochaites), and 9 lines from H27 - PKM1 x EC519791 (S. hirsutum) were screened under natural field condition. In order to create favourable environment for PBNV infection, the most susceptible host plant cowpea var. Co $(\mathrm{Cp})-7$ was planted between every two rows of tomato. No insecticide spray was given during entire crop production period. The disease incidence was assessed on $30^{\text {th }}, 45^{\text {th }}, 60^{\text {th }}$ and $90^{\text {th }}$ day after transplanting based on Percent Disease Incidence (PDI \%) and disease reaction scale. The results revealed that, PBNV disease infection was seen in all the stages of crop growth. Among the four interspecific hybrids, maximum degree of field resistance was observed in 3 lines in., H6 - Arka Vikas x EC519809 (S. peruvianum) followed by 2 lines in H4 - Arka Vikas x PSR11668, 2 lines in H17 - CO3 x EC519791 and 1 line in H27 - PKM-1 x EC519791. These 8 resistance lines will be further screened under artificial condition and field condition for confirming the resistance to PBNV and the plants showing resistance will be released as PBNV resistant variety or it may be used as a source for PBNV resistance in tomato breeding programmes.

\section{Introduction}

India is the second largest producer of vegetables in the world, next to china. Among the vegetable crops, tomato (Solanum lycopersicum. L) is the third largest cultivated vegetable crop in tropical and sub tropical regions of our country. Though the area under 
tomato cultivation is high, the production of tomato is low due to the prevalence of major biotic factors like pest and diseases. Among the diseases, viral disease plays an important role in reducing productivity. Tomato is susceptible to over 40 different groups of viruses (Allen and Gibbs. 1990). Of which, Peanut bud necrosis virus (PBNV) belongs to the member of genus Tospovirus serogroup IV, family Bunyaviridae, is a most economical and destructive plant virus affecting tomato. The incidence of tospovirus on vegetable crops especially tomato is increasing year by year (Krishna Reddy et al., 1997). The first report on occurrence of tospovirus in tomato was recorded at Australia in 1915, where as in India the occurrence was first noticed in 1964 at Nilgiris, Tamil Nadu (Todd et al., 1975). The disease is exclusively vectored by Thrips palmi Karny, in a circulative and propagative manner and transmitted in natural condition (Reddy et al., 1983). The host range of PBNV comprises of Agricultural crops, Horticultural crops and Ornamental crop. In Horticultural crops, it infects five different family of vegetables crops namely Solanaceae, Fabaceae, Cucurbitaceae, Amaranthaceae and Chenopodaceae (Umamaheswaran et al., 2003). The characteristic symptom of tospovirus on tomato are necrosis of young growing bud, bronzing of leaves with brown necrotic lesions, followed by wilting of the plant in severe cases and the ripened fruit exhibit circular markings as concentric band of red and yellow broken rings of about one $\mathrm{cm}$ in diameter (Todd et al., 1975). In tomato, the PBNV disease occurrence takes place throughout the year but the severity depends on the stage of the crop, season, location and can cause yield loss up-to 80 to 100 per cent (Venkata Ramana et al., 2011). The management of PBNV by adopting proper cultural practices are less effective and difficult due to wide host range for virus and vector. Besides, prophylactic spray schedule of pesticide for controlling thrips is ineffective due to the continues migration of thrips into the host plant from the surrounding area (Venkata Ramana et al., 2001; Kesmala et al., 2006). Generally wild relatives are renowned for their useful genes towards resistance to TSWV. Samuel et al., (1930) identified the source of resistance for TSWV for the first time in $S$. pimpinellifolium and several other sources of resistance was recorded in $S$. peruvianum, and $S$. hirsutum. By utilizing these resistance sources in tomato breeding programs, it is possible to develop resistant hybrids against $\mathrm{PBNV}$ which is an effective way to overcome PBNV infection. With the above background the study is undertaken to develop resistance in tomato against PBNV through interspecific hybridization programs.

\section{Materials and Methods}

Field screening of interspecific recombinant lines for peanut bud necrosis virus (PBNV)

The materials used in this study were initially collected from NBPGR, New Delhi and IIHR, Bangalore. In $F_{1}$ generation, thirty crosses were screened for PBNV resistance under natural field condition. Out of thirty crosses, four hybrids showed resistance to PBNV in both field screening and artificial screening through ELISA at Horticulture College and Research Institute, TNAU Coimbatore, these four crosses were further advanced to $F_{2}$ generation for field screening. The 35 single plant progenies selected in $\mathrm{F}_{3}$ from four crosses were advanced to $\mathrm{F}_{3}$ generation as detailed in Table 1. The present research was carried out at the Department of Horticulture, Agricultural College and Research Institute, Tamil Nadu Agricultural University, Madurai, Tamil Nadu, India during August to December 2019. The experimental site comprises of warm tropical climate with sand 
loamy soil, which is located at an altitude of $158 \mathrm{~m}$ above MSL between $09^{\circ} 58^{\prime} 30.5^{\prime} \mathrm{N}$ latitude and $078^{\circ} 12^{\prime} 27.4 \mathrm{E}$ longitudes.

Tomato seedlings were sown in protrays under insect proof net and standard nursery practices were followed without any application of pesticides. When the tomato seedlings attained 25 days old, they were transplanted in the main field with a spacing of $30 \times 30 \mathrm{~cm}$. For every two rows of tomato, one row of cowpea var. Co (CP) - 7 was planted which acted as a host crop and create favorable environment for PBNV. Recommended package of practices were followed as given in crop production guide, TNAU and no pesticide was sprayed during the entire crop production period to facilitate the natural field infection of PBNV. The disease incidence was assessed on 30, 45, 60, 90 days after planting based on Per-cent disease incidence, PDI $(\%)=$ Number of plants affected with PBNV / Total number of plants X 100 (Salam et al., 2011) and disease scoring was categorized into six different disease rating grade as suggested by Joi and Summanwar, 1986) (Table 2) The data on per-cent disease incidence was arcsinetransformed and statistically analysed.

\section{Results and Discussion}

Totally 35 interspecific tomato hybrids derivatives from four crosses viz., eight lines from H4- Arka Vikas x PSR11668 $(S$. pimpinellifolium), ten lines from H6 - Arka Vikas x EC519809 (S. peruvianum), eight lines from H17 - CO3 x EC519791 ( $S$. habrochaites) and nine lines from H27 PKM1 x EC519791 (S. hirsutum) were screened for PBNV resistance under field condition and disease reaction was calculated using Per-cent Disease Incidence (PDI \%) (Table.3).

Table.1 Details of $\mathrm{F}_{3}$ generation materials are given below

\begin{tabular}{|l|l|l|c|}
\hline Sl. No & \multicolumn{1}{|c|}{ Name of Interspecific Hybrids } & \multicolumn{1}{|c|}{ Selected plants in $\mathbf{F}_{\mathbf{2}}$} & Total lines raise in $\mathbf{F}_{\mathbf{3}}$ \\
\hline 1. & H4 - Arka Vikas x PSR11668 (S. pimpinellifolium) & $10,13,25,43,99,105$, & 08 \\
\hline 2. & H6 - Arka Vikas x EC519809 (S. peruvianum) & $\begin{array}{l}115,120 . \\
12,48,53,71,77,100,\end{array}$ & 10 \\
\hline 3. & H17 - CO3 x EC519791 (S. habrochaites) & $\begin{array}{l}148,152,169,212 . \\
110,141 .\end{array}$ & 08 \\
\hline 4. & H27 - PKM1 x EC519791 (S. hirsutum) & $66,88,91,112,122$, & 09 \\
\hline
\end{tabular}

Table.2 Disease reaction scale in tomato for PBNV

\begin{tabular}{|c|c|l|l|}
\hline Grade & PDI per cent & \multicolumn{1}{|c|}{ Category } & \multicolumn{1}{c|}{ Symptoms } \\
\hline A & 0.00 & Immune (I) & No symptoms \\
\hline B & 0.1 to 5.00 & Resistant (R) & Initial symptoms on younger leaflets \\
\hline C & 5.1 to 10.00 & Moderately Resistant (MR) & Symptoms extended up to petiole \\
\hline D & 10.1 to 15.0 & Moderately susceptible (MS) & Necrosis in growing branches and buds \\
\hline E & 15.1 to 25.0 & Susceptible (S) & $\begin{array}{l}\text { Necrosis extended up to stem and covering } \\
\text { entire plant }\end{array}$ \\
\hline F & 25.1 and above & Highly susceptible (HS) & Severe necrosis and wilting \\
\hline
\end{tabular}


Table.3 Detail PDI (\%) for the selected lines in $\mathrm{F}_{3}$ generation

\begin{tabular}{|c|c|c|c|c|c|c|c|}
\hline \multirow[t]{2}{*}{ Sl. No } & \multirow[t]{2}{*}{ Lines } & \multicolumn{4}{|c|}{ Per cent disease incidence (PDI) } & \multirow[t]{2}{*}{ Mean PDI \% } & \multirow{2}{*}{$\begin{array}{l}\text { Disease } \\
\text { reaction }\end{array}$} \\
\hline & & $\begin{array}{l}\text { 30th } \\
\text { day }\end{array}$ & $\begin{array}{l}\text { 45th } \\
\text { day }\end{array}$ & $\begin{array}{l}\text { 60th } \\
\text { day }\end{array}$ & $\begin{array}{l}\text { 90th } \\
\text { day }\end{array}$ & & \\
\hline \multicolumn{8}{|c|}{ H4 - Arka Vikas x PSR11668 (S. pimpinellifolium) } \\
\hline 1 & H4-10 & $\begin{array}{c}0.00 \\
(0.00)\end{array}$ & $\begin{array}{c}0.00 \\
(0.00)\end{array}$ & $\begin{array}{c}06.66 \\
(14.96)\end{array}$ & $\begin{array}{c}13.33 \\
(21.41)\end{array}$ & $\begin{array}{c}04.99 \\
(12.91)\end{array}$ & $\mathrm{R}$ \\
\hline 2 & $\mathrm{H} 4-13$ & $\begin{array}{c}0.00 \\
(0.00)\end{array}$ & $\begin{array}{c}12.5 \\
(20.70)\end{array}$ & $\begin{array}{c}12.50 \\
(20.70)\end{array}$ & $\begin{array}{c}25.00 \\
(30.00)\end{array}$ & $\begin{array}{c}12.50 \\
(20.70)\end{array}$ & MS \\
\hline 3 & $\mathrm{H} 4-25$ & $\begin{array}{c}5.26 \\
(13.26)\end{array}$ & $\begin{array}{c}15.78 \\
(23.41)\end{array}$ & $\begin{array}{c}15.78 \\
(23.41)\end{array}$ & $\begin{array}{c}21.05 \\
(27.31)\end{array}$ & $\begin{array}{c}14.46 \\
(22.35)\end{array}$ & MS \\
\hline 4 & $\mathrm{H} 4-43$ & $\begin{array}{c}0.00 \\
(0.00)\end{array}$ & $\begin{array}{c}6.25 \\
(14.48)\end{array}$ & $\begin{array}{c}06.25 \\
(14.48)\end{array}$ & $\begin{array}{c}25.00 \\
(30.00)\end{array}$ & $\begin{array}{c}09.37 \\
(17.82)\end{array}$ & MR \\
\hline 5 & H4-99 & $\begin{array}{c}0.00 \\
(0.00)\end{array}$ & $\begin{array}{c}0.00 \\
(0.00)\end{array}$ & $\begin{array}{c}03.33 \\
(10.51)\end{array}$ & $\begin{array}{c}66.66 \\
(54.73)\end{array}$ & $\begin{array}{c}17.49 \\
(24.72)\end{array}$ & $\mathrm{S}$ \\
\hline 6 & H4-105 & $\begin{array}{c}0.00 \\
(0.00)\end{array}$ & $\begin{array}{c}0.00 \\
(0.00)\end{array}$ & $\begin{array}{c}0.00 \\
(0.00)\end{array}$ & $\begin{array}{c}40.00 \\
(39.23)\end{array}$ & $\begin{array}{c}10.00 \\
(18.43)\end{array}$ & MR \\
\hline 7 & H4-115 & $\begin{array}{c}0.00 \\
(0.00)\end{array}$ & $\begin{array}{c}0.00 \\
(0.00)\end{array}$ & $\begin{array}{c}07.69 \\
(16.10)\end{array}$ & $\begin{array}{c}07.69 \\
(16.10)\end{array}$ & $\begin{array}{c}03.84 \\
(11.30)\end{array}$ & $\mathrm{R}$ \\
\hline 8 & H4-120 & $\begin{array}{c}3.92 \\
(11.42)\end{array}$ & $\begin{array}{c}9.80 \\
(18.24)\end{array}$ & $\begin{array}{c}17.64 \\
(24.83)\end{array}$ & $\begin{array}{c}21.56 \\
(27.67)\end{array}$ & $\begin{array}{c}13.23 \\
(21.33)\end{array}$ & MS \\
\hline \multicolumn{2}{|c|}{ SEd } & $\begin{array}{c}0.71 \\
(1.90)\end{array}$ & $\begin{array}{c}2.15 \\
(3.49)\end{array}$ & $\begin{array}{c}2.02 \\
(2.63)\end{array}$ & $\begin{array}{c}6.09 \\
(3.90)\end{array}$ & $\begin{array}{c}1.54 \\
(1.53)\end{array}$ & - \\
\hline \multicolumn{2}{|c|}{$\begin{array}{c}\text { CD } \\
(P \leq \mathbf{0 . 0 5})\end{array}$} & $\begin{array}{c}1.47 \\
(3.91)\end{array}$ & $\begin{array}{c}4.42 \\
(7.20) \\
\end{array}$ & $\begin{array}{c}4.15 \\
(5.42)\end{array}$ & $\begin{array}{l}12.54 \\
(8.03)\end{array}$ & $\begin{array}{c}3.17 \\
(\mathbf{3 . 1 5}) \\
\end{array}$ & - \\
\hline \multicolumn{8}{|c|}{ H6 - Arka Vikas x EC519809 (S. peruvianum) } \\
\hline 1 & H6-42 & $\begin{array}{c}0.00 \\
(0.00)\end{array}$ & $\begin{array}{c}9.09 \\
(17.55)\end{array}$ & $\begin{array}{c}09.09 \\
(17.55)\end{array}$ & $\begin{array}{c}27.27 \\
(31.48)\end{array}$ & $\begin{array}{c}11.36 \\
(19.70)\end{array}$ & MS \\
\hline 2 & H6-48 & $\begin{array}{c}0.00 \\
(0.00)\end{array}$ & $\begin{array}{c}0.00 \\
(0.00)\end{array}$ & $\begin{array}{c}4.34 \\
(12.02)\end{array}$ & $\begin{array}{c}15.21 \\
(22.95)\end{array}$ & $\begin{array}{c}4.88 \\
(12.76)\end{array}$ & $\mathrm{R}$ \\
\hline 3 & H6-53 & $\begin{array}{c}4.65 \\
(12.45)\end{array}$ & $\begin{array}{c}4.65 \\
(12.45)\end{array}$ & $\begin{array}{c}11.62 \\
(19.93)\end{array}$ & $\begin{array}{c}16.27 \\
(23.79)\end{array}$ & $\begin{array}{c}9.29 \\
(17.75)\end{array}$ & MR \\
\hline 4 & H6-71 & $\begin{array}{c}3.84 \\
(11.30)\end{array}$ & $\begin{array}{c}3.84 \\
(11.30)\end{array}$ & $\begin{array}{c}11.53 \\
(19.85)\end{array}$ & $\begin{array}{c}15.38 \\
(23.09)\end{array}$ & $\begin{array}{c}8.64 \\
(17.09)\end{array}$ & MR \\
\hline 5 & H6-77 & $\begin{array}{c}0.00 \\
(0.00)\end{array}$ & $\begin{array}{c}6.66 \\
(14.96)\end{array}$ & $\begin{array}{c}11.11 \\
(19.47)\end{array}$ & $\begin{array}{c}20.00 \\
(26.57)\end{array}$ & $\begin{array}{c}9.44 \\
(17.89)\end{array}$ & MR \\
\hline 6 & H6-100 & $\begin{array}{c}0.00 \\
(0.00)\end{array}$ & $\begin{array}{c}0.00 \\
(0.00)\end{array}$ & $\begin{array}{c}5.66 \\
(13.76)\end{array}$ & $\begin{array}{c}11.32 \\
(19.66)\end{array}$ & $\begin{array}{c}4.24 \\
(11.88)\end{array}$ & $\mathrm{R}$ \\
\hline 7 & H6-148 & $\begin{array}{c}0.00 \\
(0.00)\end{array}$ & $\begin{array}{c}0.00 \\
(0.00)\end{array}$ & $\begin{array}{c}25.00 \\
(30.00)\end{array}$ & $\begin{array}{c}50.00 \\
(45.00)\end{array}$ & $\begin{array}{c}18.75 \\
(25.66)\end{array}$ & S \\
\hline 8 & H6-152 & $\begin{array}{c}0.00 \\
(0.00)\end{array}$ & $\begin{array}{c}0.00 \\
(0.00)\end{array}$ & $\begin{array}{c}13.33 \\
(21.41)\end{array}$ & $\begin{array}{c}46.66 \\
(43.08)\end{array}$ & $\begin{array}{c}14.99 \\
(22.78)\end{array}$ & MS \\
\hline 9 & H6-169 & $\begin{array}{c}0.00 \\
(0.00)\end{array}$ & $\begin{array}{c}13.33 \\
(21.41)\end{array}$ & $\begin{array}{c}13.33 \\
(21.41)\end{array}$ & $\begin{array}{c}33.33 \\
(35.26)\end{array}$ & $\begin{array}{c}14.99 \\
(22.78)\end{array}$ & MS \\
\hline 10 & H6-212 & $\begin{array}{c}0.00 \\
(0.00)\end{array}$ & $\begin{array}{c}0.00 \\
(0.00)\end{array}$ & $\begin{array}{c}0.00 \\
(0.00)\end{array}$ & $\begin{array}{c}20.00 \\
(26.57)\end{array}$ & $\begin{array}{c}5.00 \\
(12.92)\end{array}$ & $\mathrm{R}$ \\
\hline \multicolumn{2}{|c|}{ SEd } & 0.54 & 1.41 & 2.01 & 4.08 & 1.47 & - \\
\hline
\end{tabular}




\begin{tabular}{|c|c|c|c|c|c|c|c|}
\hline \multirow{2}{*}{\multicolumn{2}{|c|}{$\begin{array}{c}\text { CD } \\
(P \leq 0.05)\end{array}$}} & (1.50) & (2.59) & (2.35) & $(2.63)$ & (1.41) & \\
\hline & & $\begin{array}{c}1.11 \\
(3.10)\end{array}$ & $\begin{array}{c}2.91 \\
(5.33)\end{array}$ & $\begin{array}{c}4.13 \\
(4.84)\end{array}$ & $\begin{array}{c}8.41 \\
(5.42)\end{array}$ & $\begin{array}{c}3.02 \\
(2.90)\end{array}$ & - \\
\hline \multicolumn{8}{|c|}{ H17 - CO3 x EC519791(S. habrochaites) } \\
\hline 1 & $\mathrm{H} 17-2$ & $\begin{array}{c}0.00 \\
(0.00)\end{array}$ & $\begin{array}{c}3.70 \\
(11.09)\end{array}$ & $\begin{array}{c}3.70 \\
(11.09)\end{array}$ & $\begin{array}{c}11.32 \\
(19.66)\end{array}$ & $\begin{array}{c}4.68 \\
(12.49)\end{array}$ & $\mathrm{R}$ \\
\hline 2 & H17-18 & $\begin{array}{c}3.12 \\
(10.17)\end{array}$ & $\begin{array}{c}3.12 \\
(10.17)\end{array}$ & $\begin{array}{c}9.37 \\
(17.82)\end{array}$ & $\begin{array}{c}21.87 \\
(27.88)\end{array}$ & $\begin{array}{c}9.37 \\
(17.82)\end{array}$ & MR \\
\hline 3 & H17-28 & $\begin{array}{c}0.00 \\
(0.00)\end{array}$ & $\begin{array}{c}5.88 \\
(14.03)\end{array}$ & $\begin{array}{c}11.76 \\
(20.06)\end{array}$ & $\begin{array}{c}23.52 \\
(29.01)\end{array}$ & $\begin{array}{c}10.29 \\
(18.71)\end{array}$ & MS \\
\hline 4 & H17-38 & $\begin{array}{c}0.00 \\
(0.00)\end{array}$ & $\begin{array}{c}0.00 \\
(0.00)\end{array}$ & $\begin{array}{c}11.76 \\
(20.06)\end{array}$ & $\begin{array}{c}17.64 \\
(24.83)\end{array}$ & $\begin{array}{c}7.39 \\
(15.77)\end{array}$ & MR \\
\hline 5 & H17-44 & $\begin{array}{c}0.00 \\
(0.00)\end{array}$ & $\begin{array}{c}0.00 \\
(0.00)\end{array}$ & $\begin{array}{c}5.00 \\
(12.92)\end{array}$ & $\begin{array}{c}15.00 \\
(22.79)\end{array}$ & $\begin{array}{c}5.00 \\
(12.92)\end{array}$ & $\mathrm{R}$ \\
\hline 6 & H17-63 & $\begin{array}{c}10.00 \\
(18.43)\end{array}$ & $\begin{array}{c}10.00 \\
(18.43)\end{array}$ & $\begin{array}{c}30.00 \\
(33.21)\end{array}$ & $\begin{array}{c}40.00 \\
(39.23)\end{array}$ & $\begin{array}{c}22.5 \\
(28.32)\end{array}$ & $\mathrm{S}$ \\
\hline 7 & H17-110 & $\begin{array}{c}7.40 \\
(15.79)\end{array}$ & $\begin{array}{c}11.11 \\
(19.47)\end{array}$ & $\begin{array}{c}11.11 \\
(19.47)\end{array}$ & $\begin{array}{c}22.22 \\
(28.12)\end{array}$ & $\begin{array}{c}12.96 \\
(21.10)\end{array}$ & MS \\
\hline 8 & H17-141 & $\begin{array}{c}4.76 \\
(12.60)\end{array}$ & $\begin{array}{c}4.76 \\
(12.60)\end{array}$ & $\begin{array}{c}9.52 \\
(17.97)\end{array}$ & $\begin{array}{c}23.80 \\
(29.20)\end{array}$ & $\begin{array}{c}10.71 \\
(19.10)\end{array}$ & MS \\
\hline \multicolumn{2}{|c|}{ SEd } & $\begin{array}{c}1.29 \\
(2.64)\end{array}$ & $\begin{array}{c}1.36 \\
(2.44)\end{array}$ & $\begin{array}{c}2.66 \\
(2.19)\end{array}$ & $\begin{array}{c}2.83 \\
(1.91)\end{array}$ & $\begin{array}{c}1.88 \\
(1.67)\end{array}$ & - \\
\hline \multicolumn{2}{|c|}{$\begin{array}{c}\text { CD } \\
(P \leq 0.05)\end{array}$} & $\begin{array}{c}2.67 \\
(5.43)\end{array}$ & $\begin{array}{c}2.80 \\
(5.03)\end{array}$ & $\begin{array}{c}5.49 \\
(4.51)\end{array}$ & $\begin{array}{c}5.82 \\
(3.94)\end{array}$ & $\begin{array}{c}3.86 \\
(3.44)\end{array}$ & - \\
\hline \multicolumn{8}{|c|}{ H27 - PKM1 x EC519791 (S. hirsutum) } \\
\hline 1 & H27-66 & $\begin{array}{c}2.59 \\
(9.26)\end{array}$ & $\begin{array}{c}6.49 \\
(14.76)\end{array}$ & $\begin{array}{c}9.09 \\
(17.55)\end{array}$ & $\begin{array}{c}16.88 \\
(24.26)\end{array}$ & $\begin{array}{c}8.76 \\
(17.22)\end{array}$ & MR \\
\hline 2 & $\mathrm{H} 27-88$ & $\begin{array}{c}9.15 \\
(17.61)\end{array}$ & $\begin{array}{c}12.08 \\
(20.34)\end{array}$ & $\begin{array}{c}16.48 \\
(23.95)\end{array}$ & $\begin{array}{c}21.97 \\
(27.95)\end{array}$ & $\begin{array}{c}14.92 \\
(22.72)\end{array}$ & MS \\
\hline 3 & H27-91 & $\begin{array}{c}3.28 \\
(10.43)\end{array}$ & $\begin{array}{c}5.92 \\
(14.08)\end{array}$ & $\begin{array}{c}7.89 \\
(16.31)\end{array}$ & $\begin{array}{c}15.13 \\
(22.89)\end{array}$ & $\begin{array}{c}8.05 \\
(16.48)\end{array}$ & MR \\
\hline 4 & $\mathrm{H} 27-112$ & $\begin{array}{c}7.69 \\
(16.10)\end{array}$ & $\begin{array}{c}9.61 \\
(18.06)\end{array}$ & $\begin{array}{c}17.30 \\
(24.58)\end{array}$ & $\begin{array}{c}23.07 \\
(28.71)\end{array}$ & $\begin{array}{c}14.41 \\
(22.31)\end{array}$ & MS \\
\hline 5 & H27-122 & $\begin{array}{c}3.29 \\
(10.45)\end{array}$ & $\begin{array}{c}5.49 \\
(13.55)\end{array}$ & $\begin{array}{c}15.38 \\
(23.09)\end{array}$ & $\begin{array}{c}24.17 \\
(29.45)\end{array}$ & $\begin{array}{c}12.08 \\
(20.34)\end{array}$ & MS \\
\hline 6 & H27-168 & $\begin{array}{c}8.13 \\
(16.57)\end{array}$ & $\begin{array}{c}12.19 \\
(20.43)\end{array}$ & $\begin{array}{c}17.07 \\
(24.40)\end{array}$ & $\begin{array}{c}23.57 \\
(29.04)\end{array}$ & $\begin{array}{c}15.24 \\
(22.98)\end{array}$ & $\mathrm{S}$ \\
\hline 7 & $\mathrm{H} 27-173$ & $\begin{array}{c}0.00 \\
(0.00)\end{array}$ & $\begin{array}{c}3.70 \\
(11.09)\end{array}$ & $\begin{array}{c}5.92 \\
(14.08)\end{array}$ & $\begin{array}{c}10.37 \\
(18.79)\end{array}$ & $\begin{array}{c}4.99 \\
(12.91)\end{array}$ & $\mathrm{R}$ \\
\hline 8 & H27-218 & $\begin{array}{c}9.21 \\
(17.67)\end{array}$ & $\begin{array}{c}12.76 \\
(20.93)\end{array}$ & $\begin{array}{c}19.14 \\
(25.94)\end{array}$ & $\begin{array}{c}24.11 \\
(29.41)\end{array}$ & $\begin{array}{c}16.30 \\
(23.81)\end{array}$ & S \\
\hline 9 & $\mathrm{H} 27-212$ & $\begin{array}{c}0.00 \\
(0.00)\end{array}$ & $\begin{array}{c}7.69 \\
(16.10)\end{array}$ & $\begin{array}{c}12.30 \\
(20.53)\end{array}$ & $\begin{array}{c}26.15 \\
(30.76)\end{array}$ & $\begin{array}{c}11.53 \\
(19.85)\end{array}$ & MS \\
\hline \multicolumn{2}{|c|}{ SEd } & $\begin{array}{c}1.18 \\
(2.02)\end{array}$ & $\begin{array}{c}1.05 \\
(1.11)\end{array}$ & $\begin{array}{c}1.50 \\
(1.33)\end{array}$ & $\begin{array}{c}1.65 \\
(1.24)\end{array}$ & $\begin{array}{c}1.21 \\
(1.15)\end{array}$ & - \\
\hline \multicolumn{2}{|c|}{$\begin{array}{c}\text { CD } \\
(P \leq 0.05)\end{array}$} & $\begin{array}{c}2.44 \\
(4.52)\end{array}$ & $\begin{array}{c}2.16 \\
(2.28)\end{array}$ & $\begin{array}{c}3.08 \\
(2.74)\end{array}$ & $\begin{array}{c}3.40 \\
(2.56)\end{array}$ & $\begin{array}{c}2.49 \\
(2.36)\end{array}$ & - \\
\hline
\end{tabular}

** Non bracket- indicate original PDI value, ()- indicate arcsine- transformed PDI value, SEd- standard error difference, $\mathrm{CD}$ - critical value at 0.05 significant probability level 
The result revealed that, disease incidence was seen in all the stages of crop growth but the maximum incidence occurred at $90^{\text {th }}$ day after transplanting. However, from H6 - Arka Vikas x EC519809 (S. peruvianum) cross, 3 lines viz., H6-48, H6-100, and H6-212 were resistant, H6-53, H6-71 and $\mathrm{H6} 6-77$ are moderately resistant, followed by H6-42, H6152 and H6-169 which are moderately susceptible and the line H6-148 was susceptible to PBNV infection. From the cross, H4 - Arka Vikas x PSR11668 (S. pimpinellifolium), out of 8 lines screened for PBNV resistance, the lines H4-10 and H4-115 showed resistance reaction to PBNV infection, H4-43, and H4-105 are moderately resistant followed by the lines H4-13, H4-25 and H4-120 which showed moderately susceptible reaction and the line H4-99 was susceptible to PBNV infection. From the cross H17 - CO3 x EC519791 ( $S$. habrochaites), out of 8 lines screened for PBNV resistance, the line H17-2 and H17-44 showed resistance to PBNV infection and the lines H17-18, and H17-38 showed moderately resistant reaction and the lines H17-28, H17110 , and H17-141 were moderately susceptible followed by the line H17-63 which was susceptible to PBNV infection. In the cross H27 - PKM1 x EC519791 ( $S$. hirsutum), out of 9 lines screened for PBNV resistance, the line H27-173 showed resistance to PBNV infection, H27-66, and H27-91 are moderately resistant followed by the lines $\mathrm{H} 27-88, \mathrm{H} 27-112, \mathrm{H} 27-122$, and H27-212 which are moderately susceptible and H27-168 and H27-218 are susceptible to PBNV infection.

The present results were similar with the findings of Gordillo et al., 2008, Paterson et al., 1989, Rosello et al., 1997, Satish Sain and Chadha, 2016. These 8 lines showing resistance to PBNV infection will be further screened under artificial and field condition for confirming the resistance to PBNV and the plants may be used as a source for PBNV resistance in tomato breeding programmes.

\section{References}

Allen, B, and Gibbs, K. C. 1990. Viruses of tropical plants. CAB International, Oxon, UK. 707pp.

Gordillo, L.F., Stevens, M.R., Millard, M.A. and Geary, B., 2008. Screening two Lycopersicon peruvianum collections for resistance to Tomato spotted wilt virus. Plant disease, 92(5), pp.694704.

Joi, M.B. and Summanvar, A.S., 1986. Occurrence of tomato spotted wilt virus on tomato in Maharashatra. Maharashtra J. Hort, 3, pp.68-72.

Kesmala, T., Jogloy, S., Wongkaew, S., Akkasaeng, C. and Patanothai, A., 2006. Evaluation of ten peanut genotypes for resistance to Peanut bud necrosis virus (PBNV). Evaluation, $28(3)$, p. 460.

Krishna Kumar, N.K., Ullman, D.E. and Cho, J.J., 1993. Evaluation of Lycopersicon germ plasm for tomato spotted wilt tospovirus resistance by mechanical and thrips transmission. Plant disease, 77(9), pp.938-941.

Krishna Reddy, M., Gopal, K., Akelavani and Sigh, S. J., 1997. Immunological detection and serological comparison of tospovirus isolated using polyclonal and monoclonal antibodies. In: International conference on Integrated Plant Disease Management for Sustainable Agriculture, IPS golden jubilee, IARI, New Delhi November 10-15, p36

Paterson, R.G., Scott, S.J. and Gergerich, R.C., 1989. Resistance in two Lycopersicon species to an Arkansas isolate of tomato spotted wilt virus. Euphytica, 43(1-2), pp.173-178.

Reddy, D.V.R., Amin, P.W., McDonald, D. 
and Ghanekar, A.M., 1983. Epidemiology and control of groundnut bud necrosis and other diseases of legume crops in India caused by tomato spotted wilt virus.

Roselló, S., Díez, M.J., Lacasa, A., Jordá, C. and Nuez, F., 1997. Testing resistance to TSWV introgressed from Lycopersicon peruvianum by artificial transmission techniques. Euphytica, 98(1-2), pp.93-98.

Salam, S.A., Patil, M.S. and Dyadgi, A.S., 2011. Status of mungbean yellow mosaic virus disease incidence on green gram. Karnataka Journal of Agricultural Sciences, 24(2).

Samuel, G., Bald, J.G. and Pittman, H.A., 1930. Investigations on" Spotted Wilt" of Tomatoes. Bulletin of the Council for Scientific and Industrial Research, Australia, (44).

Sain, S.K. and Chadha, M.L., 2016. Response of some wild species of tomato against Peanut bud necrosis virus under open- field conditions. Indian Journal of Horticulture, 73(1), pp.60-65.

Todd, J.M., Ponniah, S. and Subramanyam, C.P., 1975. First record of tomato spotted wilt virus from the Nilgirisin India. Madras agricultural journal.

Umamaheswaran, K., Jain, R.K., Bhat, A.I. and Ahlawat, Y.S., 2003. Biological and molecular characterization of a Tospovirus isolate from tomato and its relationship with other Tospoviruses. Indian Phytopathology, 56(2), pp.168173.

Venkata Ramana, C., Venkata Rao, P., Prasada Rao, R.D.V.J., Kumar, S.S., Reddy, I.P. and Reddy, Y.N., 2010, July. Genetic analysis for Peanut bud necrosis virus (PBNV) resistance in tomato (Lycopersicon esculentum Mill.). In III International Symposium on Tomato Diseases 914 (pp. 459463).

\section{How to cite this article:}

Arumugam Nithyanandam, A. Beaulah, S. Harish, C. Rajamanickam, S. Vellaikumar and Arunachalam, P. 2020. Screening of $\mathrm{F}_{3}$ Generation Interspecific Tomato Hybrids Derivatives for Resistance to Peanut Bud Necrosis Virus (PBNV). Int.J.Curr.Microbiol.App.Sci. 9(08): 1431-1437. doi: https://doi.org/10.20546/ijcmas.2020.908.164 